\title{
REVIEWS
}

\section{The influence of ageing on the development and management of rheumatoid arthritis}

\author{
Annemieke M. H. Boots, Andrea B. Maier, Piet Stinissen, Pierre Masson, Rik J. Lories and Filip De Keyser
}

\begin{abstract}
The population of elderly individuals with rheumatoid arthritis (RA) is expanding, due mainly to increasing life expectancy. A variety of theories have been proposed to explain the ageing process, including accumulation of DNA damage and resultant changes in biological processes. Such changes can influence the development and/or course of disease. Furthermore, alterations in biological function determine the biological age-as opposed to chronological age — of an individual, which strongly influences their ability to cope with disease. Moreover, comorbidities are more frequent in elderly individuals. Together, these factors complicate treatment of disease and necessitate careful patient management. Indeed, although evidence from clinical trials suggests that DMARDs and biologic agents have good efficacy and are well tolerated in elderly patients with RA, such individuals are often undertreated and inadequately managed. Unfortunately, insufficient data are available for the development of evidence-based guidelines for this population, as elderly patients are often excluded from clinical trials owing to age restrictions or comorbidities. Thus, additional clinical studies in elderly patients are warranted, with treatment regimens tailored according to vitality or frailty parameters. This Review focuses on the pathophysiological aspects of ageing and their implications for the management of RA in elderly patients.
\end{abstract}

Boots, A. M. H. et al. Nat. Rev. Rheumatol. 9, 604-613 (2013); published online 18 June 2013; doi:10.1038/nrrheum.2013.92

\section{Introduction}

Evidence-based treatment recommendations for rheumatoid arthritis (RA) have been developed mostly for middle-aged patients and have not been validated for elderly patients (considered as those aged $\geq 65$ years unless otherwise stated). However, as life expectancy increases, the number of elderly patients with RA is increasing, and thus new strategies are needed to optimize therapy in this population. Limited data are available regarding the treatment of RA in individuals aged 65 years and older due to age-based selection criteria or because such patients often have comorbidities, factors which restrict their inclusion in intervention studies. ${ }^{1}$ Ageing in humans is a multidimensional process comprising changes in physical, psychological and social factors over time. Alterations in these dimensions, particularly biological changes, can affect disease susceptibility, progression and coping, as well as the effectiveness and safety of therapies. This Review introduces the general aspects of ageing that are of relevance to immune-mediated diseases and discusses the influence of ageing on the development, phenotype, and management of RA.

\section{Competing interest}

P. Masson declares an association with the following company: Abbott (Abbvie). R. J. Lories declares associations with the following companies: Abbott (Abbvie), Boehringer Ingelheim, Celgene, Jansen, Merck and Pfizer. F. De Keyser declares an association with the following companies: Abbott (Abbvie), AstraZeneca, GSK, MSD, Pfizer and Roche. See the article online for details of the relationships. A. M. H. Boots, A. B. Maier and P. Stinissen declare no competing interests.

\section{Mechanisms of ageing}

Ageing remains a mysterious process; the causes of ageing, the factors that determine the rate at which it occurs, and which changes account for the increased chance of dying beyond middle age are still unclear. Three main theories have been proposed to explain the ageing process: the molecular phenomena theory, the disposable soma theory, and the evolutionary theory. The theories are not necessarily mutually exclusive, and no particular hypothesis can be deemed the most accurate.

\section{The molecular phenomena theory of ageing}

A variety of molecular phenomena participate in ageing at the cellular level, although accumulation of DNA damage with age is thought to be of particular importance. Chromosomal telomeres shorten with each cell division, which eventually leads to cell-cycle arrest and cellular senescence, ${ }^{2}$ thus limiting the capacity for regeneration and renewal of cells and tissues. Moreover, genotoxic stressors, such as radiation and reactive oxygen species produced during normal metabolic activity, can cause progressive accumulation of DNA damage. The quality and efficiency of the DNA repair processes themselves might also be affected and decrease with age, further compromising the integrity of the genome. ${ }^{3}$ Accumulation of DNA damage and mutations can lead to cellular damage or dysfunction-for example, unbalanced expression of oncogenes and tumour suppressor genes that results in oncogenic transformation-and/or to cell death. Together, such processes are responsible for the biological phenotype of ageing and can influence both the development and management of disease. 


\section{The disposable soma theory of ageing}

The disposable soma theory explains ageing from the perspective of developmental biology. ${ }^{4,5}$ According to this theory, ageing is the price of survival. In the body, energy must be distributed to multiple processes over time, which could lead to processes that are less important for survival of the species being compromised in favour of those deemed more important. As reproduction is the most important biological process for survival of the species, maintenance and repair processes are focused on reproductive organs and germline cells, with the function of somatic cells and other tissues declining over time as a result of insufficient repair and renewal. For example, the thymus, an organ that is essential for the development and selection of T cells, gradually involutes-whereby thymic tissue is replaced by fat-once adulthood is reached and the $\mathrm{T}$-cell repertoire has been established.

\section{The evolutionary theory of ageing}

Evolution is driven by natural selection of genetic factors that provide a benefit to survival by enabling an organism to reproduce and pass on their genes more effectively. Genetic variants that have negative effects only in older age-when the organism is likely to have already successfully reproduced and raise offspring, and might even have become infertile-are less likely to drive evolution if they have only a limited effect on the propagation of genetic material and therefore the overall survival of the species; likewise, variants that delay ageing and promote longevity will not be selected for unless they increase the overall reproductive success of the species. Thus, according to the evolutionary theory of ageing, genes that are advantageous at a younger age can become disadvantageous at an older age and contribute to the phenotype of ageing. ${ }^{6}$ For example, high levels of testosterone aid male fertility at young age, but can later lead to alopecia, which represents a decline in hair follicle function and is seen as a sign of ageing. Another example is the human immune system, which was optimized by evolution primarily at a time when life expectancies were much shorter than today. Thus, our immune system now needs to be effective for much longer than when our species was young, which poses a challenge: over many decades, the immune system must be balanced between being strong enough to defend against infections, but not so strong as to result in autoinflammation and autoimmunity. Indeed, in old age, immune processes can become detrimental, providing inadequate protection from infection or promoting autoimmunity.

\section{Successful versus unsuccessful ageing}

Biological age, an important geriatric concept, is the rate at which an individual ages in terms of their physical and mental functionality, and is indicative of their health and life expectancy. The degree of correlation between biological age and chronological age-that is, an individual's age in years - can vary widely among individuals, especially elderly individuals. ${ }^{7}$ For example, one 80 -yearold person might still have a large social network and be

\section{Key points}

- As a consequence of increasing life expectancies worldwide, the number of elderly people with rheumatoid arthritis (RA) is growing

- Evidence-based guidelines for management of RA in elderly individuals are lacking owing to insufficient clinical data

- Data suggest that elderly individuals with RA are undertreated and inadequately managed, despite DMARDs and biologic therapies being effective and seemingly well tolerated in this population

- Therapeutic decision making in elderly patients with RA needs to incorporate consideration of patient frailty and comorbidities

- The level of disease activity set as the therapeutic goal might differ according to the patient's biological age or presence of particular risk factors for infection

physically active, whereas another individual with the same chronological age but a higher biological age would have generally poor health, limited mobility and a small social network. In this context, unsuccessful ageing can be viewed as biological age exceeding chronological age. Conversely, individuals with a biological age matching or less than their chronological age would be seen as having aged successfully. Discrepancies between biological and chronological age are accounted for by 'geriatric giant conditions', comorbidities, and frailty.

\section{Geriatric giant conditions}

Isaacs ${ }^{8}$ first described geriatric giant conditions, wherein the use of the word 'giant' reflects both the large number of elderly individuals affected and the considerable effect of the conditions on an individual's independence. Geriatric giant conditions include cognitive limitations, depression, sensory limitations (particularly affecting vision and hearing), mobility restrictions, and incontinence.

\section{Comorbidities and polypharmacy}

The prevalence of comorbidity and resultant polypharmacy increases with age and adds to the risk of unsuccessful ageing; in the Netherlands, approximately $60 \%$ of 60 -year-olds have at least two health conditions, and the frequency of comorbidity increases by $10 \%$ for each additional decade of life up to 80 years of age. ${ }^{9}$ Comorbidities are associated with increased use of multiple therapeutics and, therefore, an increased risk of drug interactions. In elderly patients with RA, for example, disease management is often complicated by both nonrheumatic comorbidities, such as cardiovascular disease, diabetes mellitus and neoplastic conditions, and the coexistence of multiple rheumatic conditions, including an association between RA and severe osteoarthritis.

\section{Frailty}

Frailty, which can be considered as the opposite of vitality, adds to the risk of unsuccessful ageing, ${ }^{10}$ and thus is a concept relevant to geriatric medicine. Frailty manifests as weakness, unintentional weight loss, exhaustion, low physical activity, and slower walking speed; according to Fried and colleagues, ${ }^{11}$ an individual is considered frail if they simultaneously demonstrate three or more of these five phenotypes. Frailty might reflect a loss of functional homeostasis, which results in a reduced ability 


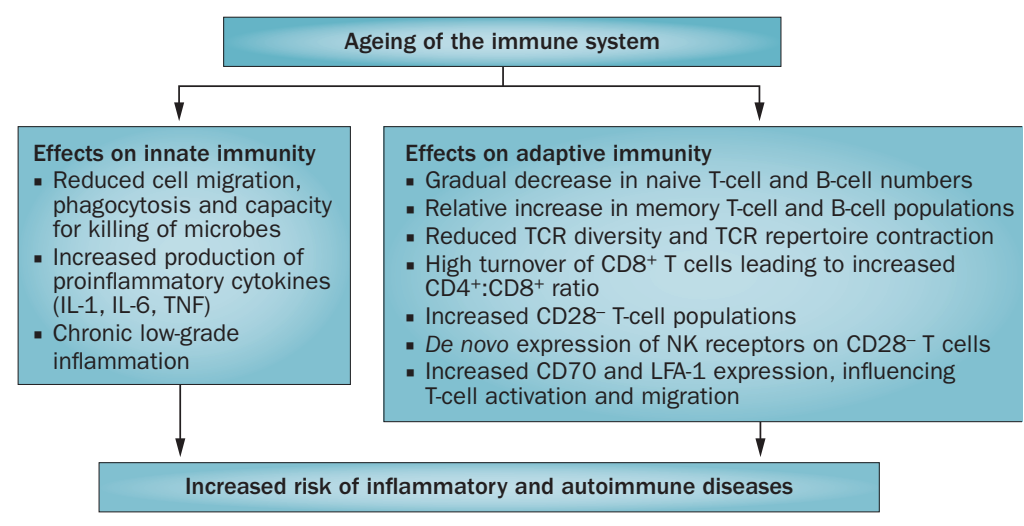

Figure 1 | Major changes of the immune system with ageing. Ageing has profound effects on both the innate and adaptive branches of the immune system: innate immune mechanisms tend to become more active, whereas functioning of the adaptive immune system generally declines. Immunosenescence is associated with a more pronounced, chronic proinflammatory activity in the innate branch of the immune system and a decrease in naive lymphocyte counts with a relative increase in memory cells in the adaptive branch. Together, these changes might contribute to the development of chronic inflammatory and autoimmune disease. Abbreviations: LFA-1, lymphocyte function-associated antigen 1 (also known as CD11a/CD18 or integrin $\alpha_{\mathrm{L}} / \beta_{2}$ ); NK, natural killer (cell); TCR, T-cell receptor.

to withstand or compensate for illness without a loss of function. For example, an 80-year-old person is less likely than a younger person to regain pre-existing functional capacity after a hip fracture, and subsequent events could lead to progressive disability and dependence. Frail individuals have an increased frequency of negative health outcomes including accidental falls, reduced mobility, decreased functional capacity, reduced efficacy of vaccination, markedly increased mortality and disability after surgery, higher rejection rates following renal transplantation, and death. ${ }^{11-15}$ Inflammatory mediators and hormones might contribute to the perpetuation of a negative cycle of frailty. ${ }^{16-18}$

\section{Changes in the immune system with age}

Ageing has profound effects on all cells and tissues of a living organism, especially those of the immune system. ${ }^{19}$ Both the innate and adaptive branches of the immune system undergo marked changes with age and contribute to the process of immunosenescence-the gradual decrease in the capacity of the immune system to resolve infection and establish long-term immunological memory (Figure 1). With age, innate immune mechanisms tend to become more active, whereas functioning of the adaptive immune system generally declines. The precise molecular and cellular mechanisms underlying this shift towards innate immunity remain largely unknown.

\section{Alterations in the innate immune system}

As the innate immune system ages, levels of TNF, IL-6, C-reactive protein (CRP) and other inflammatory molecules increase. In addition, increased numbers of natural killer (NK) cells are produced and greater amounts of proinflammatory cytokines are released by monocytes and dendritic cells. ${ }^{20-23}$ These changes create a proinflammatory environment that might accelerate development of and complicate diseases such as RA. ${ }^{24,25}$ Other ageing-related alterations in functioning of the innate immune system, such as reduced cytotoxicity of NK cells or decreased phagocytotic capacity of monocytes, might compromise the immediate response to bacterial and viral pathogens and the integration of innate and adaptive immune responses.

\section{Alterations in the adaptive immune system}

The reduced effectiveness of the adaptive immune response with ageing can be observed as decreased tuberculin skin test responses in elderly people. ${ }^{26}$ At the cellular level, ageing affects expression of B-cell markers and B-cell responses, as well as the T-cell repertoire. ${ }^{27}$ Lymphocyte numbers decrease with age, although the reduction is greater for $\mathrm{CD} 8^{+} \mathrm{T}$ cells than $\mathrm{CD} 4^{+} \mathrm{T}$ cells or $\mathrm{B}$ cells. ${ }^{28}$ This effect could be the result of a decline in the generation of novel naive $\mathrm{T}$ cells as a consequence of thymic involution and a decrease in the amount of haematopoetic tissue within the bone marrow. ${ }^{29}$ In addition, the composition of the T-cell population switches from comprising mostly naive cells to mainly effector memory and late-stage effector memory cells (Figure 2). ${ }^{30}$

Expression of CD28-a molecule that transmits important co-stimulatory signals that lead to T-cell activation and proliferation-on $\mathrm{T}$ cells is progressively lost with age. $^{31,32}$ This decline in CD28 expression is caused by the loss of a CD28-specific transcriptional initiator complex, ${ }^{33}$ as a result of repeated antigen stimulation and/or cytokine signalling, ${ }^{30,34}$ and is amplified by factors such as persistent infection with latent viruses, including cytomegalovirus. ${ }^{35,36}$ Loss of CD28 thus reflects clonal expansion of antigen-responsive T cells, which can include those responsive to self antigens. ${ }^{31} \mathrm{CD} 28^{-} \mathrm{T}$ cells do not proliferate but survive longer than their $\mathrm{CD} 28^{+}$counterparts, and have been shown to be resistant to apoptosis through expression of Bcl-2 and FLICE-like inhibitory protein (FLIP, also known as CASP8 and FADD-like apoptosis regulator). ${ }^{37,38}$ The consequences of this loss of $\mathrm{CD} 28$ seems to differ between $\mathrm{CD} 4^{+} \mathrm{T}$ cells and $\mathrm{CD} 8^{+} \mathrm{T}$ cells: in $\mathrm{CD}^{+} \mathrm{T}$ cells, the loss can lead to dysfunction or to a shift towards a regulatory phenotype,$^{32}$ whereas in $\mathrm{CD} 4^{+}$ T cells, CD28 deficiency is associated with the acquisition of novel NK-cell-like functionalities, ${ }^{39}$ possibly due to alterations in T-cell DNA methylation patterns that occur with age. ${ }^{40,41}$ Moreover, loss of CD28 in CD4 ${ }^{+} \mathrm{T}$ cells is associated with increased production of proinflammatory cytokines and a propensity to migrate into tissues. ${ }^{32}$ Interestingly, $\mathrm{CD} 4^{+} \mathrm{CD} 28^{-} \mathrm{T}$-cell populations are expanded in several chronic autoimmune diseases, including RA; ${ }^{42,43}$ repeated (auto)antigen stimulation might underlie this phenomenon. Despite the loss of CD28 associated with ageing, abatacept—a biologic agent, based on cytotoxic T-lymphocyte antigen 4, that blocks T-cell activation and is approved for the treatment of RA-does not seem to have reduced efficacy in populations of elderly patients, ${ }^{44,45}$ even though its primary mechanism of action is thought to be competitive binding to CD28 ligands and thus indirect interference with CD28 engagement and signalling. 


\section{The possible involvement of adipokines}

Changes in the levels of adipokines-cytokines that are secreted by adipose tissue - that occur with ageing could also contribute to or influence the pathogenesis of immune-mediated inflammatory disorders, including RA. Adipose-tissue dysfunction, indicated by dysregulation of adipokine expression, is associated with poor prognosis in the most elderly subset of patients with RA. ${ }^{46}$ Nevertheless, how age-related changes in adipokine levels influence both the risk and manifestation of chronic inflammatory disorders such as RA, and whether adipokines are protective or harmful in RA, are not clear. ${ }^{47,48}$ Leptin levels are generally believed to be elevated in patients with RA, ${ }^{48}$ and serum leptin levels and synovial fluid:serum leptin ratios might also correlate with duration and/or activity of disease. ${ }^{49}$ The adipokine leptin could influence RA disease processes because it can directly affect articular tissues, and can also directly modify immune-cell activation, proliferation, maturation, and production of inflammatory mediators. ${ }^{48}$ In particular, leptin can modulate the activity of regulatory $\mathrm{T}$ cells, which are potent suppressors of autoimmunity. ${ }^{50}$ Levels of adiponectin, another adipokine, are also increased in RA and correlate with disease severity. ${ }^{51}$ This association might reflect the ability of adiponectin to increase inflammation and extracellular matrix degradation within joints. ${ }^{48}$

\section{Consequences of immunosenescence}

With age, increases in susceptibility, morbidity, and mortality are observed for several infectious diseases, including influenza, pneumococcal infections (mainly involving Streptococcus pneumonia), tuberculosis, and urinary tract infections. ${ }^{52-54}$ Higher rates of infection in adults $>65$ years of age are due in part to immunosenescence and possibly to comorbidities and nutritional deficiencies. Vaccination has helped decrease the rates of infection, morbidity and mortality in elderly individuals, but this benefit has been hampered by the reduced immune responses to vaccination associated with immunosenescence in this patient population. ${ }^{55-59}$ For example, influenza vaccine efficacy is $70 \%$ to $90 \%$ for non-elderly people (aged $<65$ years) but only $17 \%$ to $53 \%$ in elderly individuals, ${ }^{55,58-59}$ and patterns are similar for tetanus, S. pneumonia, and hepatitis B. ${ }^{56,57}$ Interestingly, the lymphoid apparatus in the nose and upper airways is less affected by immunosenescence than other lymphoid tissues, ${ }^{60}$ which suggests that utilization of the nasal route could improve vaccine efficacy in elderly people.

Aside from infectious diseases, several types of tumours also become more frequent and aggressive with age, such as melanoma. ${ }^{61,62}$ Although accumulation of DNA damage could partially explain this observation, weakened immune surveillance in elderly individuals also seems to be important. ${ }^{63}$ In addition, as the immune system ages, the risk of autoreactivity and thus certain immune-mediated inflammatory diseases increases. Indeed, autoantibodies, including rheumatoid factor $(\mathrm{RF})$ and antinuclear antibodies, ${ }^{64}$ are more frequently detected in older than younger adults, and

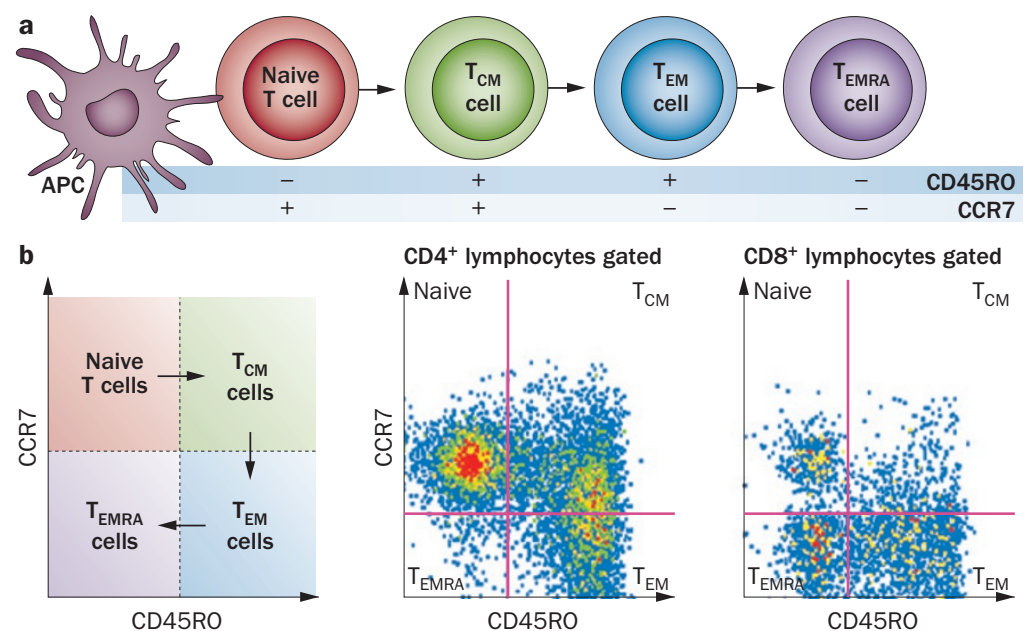

Figure 2 | Post-thymic differentiation of naive T cells after antigen presentation by professional APCs. a | Schematic representation of T-cell differentiation stages. Naive and memory T cells are required to maintain immune competence after thymic involution. Naive T cells are maintained by homeostatic proliferation, and express CCR7 and CD45RA (but not CD45RO). Upon APC-dependent antigen encounter, naive T cells are activated via TCR and CD28 signalling, and can differentiate into memory $T$ cells. Three phenotypically and functionally distinct subsets of $\mathrm{CD}^{+}$or $\mathrm{CD} 8^{+}$memory $\mathrm{T}$ cells are now recognized based on expression of CD45RO and CCR7. First, antigen-experienced, IL-2 producing $T_{C M}$ cells, which express both CD45RO and CCR7 and home to lymph nodes. Second, antigenexperienced, $\mathrm{T}_{H} 1, \mathrm{~T}_{\mathrm{H}} 2$ or $\mathrm{T}_{\mathrm{H}} 17$ (producing IFN- $\gamma$, IL 4 or IL-17, respectively) $\mathrm{T}_{\mathrm{EM}}$ cells, which express CD45RO but have lost the expression of CCR7 and thereby acquired the capacity to migrate to nonlymphoid tissues. Third, late-stage, $T_{\text {EMRA }}$ cells, which

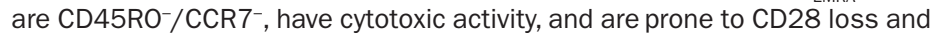
acquisition of novel functionalities usually associated with natural killer cells. b | Flow cytometry detection of these T-cell populations in a sample of peripheral blood from a healthy individual. Although absolute T-cell numbers decline with ageing, a sample from an elderly individual, analysed in the same way, would be expected to contain increased proportions of the memory $T$ cell populations $\left(T_{C M}, T_{E M}\right.$ and $T_{E M R A}$ cells) relative to naive cells. Abbreviations: APC, antigenpresenting cell; CCR7, CC chemokine receptor 7; TCR, T-cell receptor; $\mathrm{T}_{\mathrm{CM}}$, central memory $\mathrm{T}$ (cell); $\mathrm{T}_{\mathrm{EM}}$, effector memory $\mathrm{T}$ (cell); $\mathrm{T}_{\mathrm{EMRA}}, \mathrm{CD} 45 \mathrm{RA}$-reverted effector memory $\mathrm{T}$ (cell); $\mathrm{T}_{H} 1$, type $1 \mathrm{~T}$-helper (cell); $\mathrm{T}_{\mathrm{H}} 2$, type $2 \mathrm{~T}$-helper (cell); $\mathrm{T}_{\mathrm{H}} 17$, type $17 \mathrm{~T}$-helper (cell).

some immune diseases such as polymyalgia rheumatica and giant cell arteritis do not onset until after 55 years of age, but become increasingly common thereafter. ${ }^{65}$ Such phenomena might be attributable to a loss of endogenous immune-regulatory mechanisms with ageing.

\section{Immunosenescence and RA}

Although RA can occur in individuals of any age, its incidence continues to increase with age at least into the seventh decade of life and possibly beyond. ${ }^{20}$ Crowson et al. ${ }^{66}$ reported that the cumulative lifetime risk of developing RA is $<1 \%$ before 50 years of age, but greatly increases for both genders starting at approximately 60 years of age and then plateaus after 80 years of age. Interestingly, RA is characterized by accelerated ageing of the immune system, including insufficiency of telomerase activity, deficiency of DNA repair mechanisms, impaired regeneration of T cells, contracted diversity of the T-cell repertoire, and increased frequency of $\mathrm{CD} 28^{-}$ T cells. ${ }^{24,32,67,68}$ These signs of accelerated ageing of the immune system are evident in patients with early RA 
and are not influenced by duration or treatment of disease. ${ }^{42,69}$ Changes associated with accelerated ageing of the immune system could lead to the selection of a T-cell repertoire with increased self-reactivity and conversion of self-reactive naive $\mathrm{T}$ cells into memory-like cells. ${ }^{70}$

The bias towards autoreactivity in both the naive and effector peripheral T-cell repertoires in RA could be the result of unbalanced homeostatic T-cell proliferation of naive $\mathrm{T}$ cells in the periphery - the primary mechanism for replenishment of $\mathrm{T}$ cells in adults. ${ }^{71,72}$ Increased apoptosis as a consequence of deficient DNA repair ${ }^{72}$ and proliferative-stress-induced senescence resulting from telomere shortening, ${ }^{71}$ possibly secondary to telomerase insufficiency, ${ }^{67}$ might cause lymphopeniainduced T-cell proliferation and remodelling of the T-cell repertoire, which could contribute to development of autoimmunity and accelerated aging in RA. Autoreactivity is thought to arise from the peripheral homeostatic proliferation of naive $\mathrm{T}$ cells owing to positive post-thymic selection dependent on the affinity of T-cell receptors for self-peptide-MHC complexes on antigen-presenting cells. ${ }^{73}$ This positive selection of naive $\mathrm{T}$ cells implies that $\mathrm{T}$ cells with lower specificities for self-peptides do not receive sufficient survival signals and gradually disappear from the T-cell repertoire, causing it to diminish. Homeostatic proliferation in the periphery is sufficient to induce T-cell differentiation leading to the generation of late-stage effector cells (Figure 2). The accumulation of late-stage effector memory cells with proinflammatory characteristics could promote chronic inflammation and development of autoimmunity in the elderly: ${ }^{70}$ as we have alluded to previously, late-stage effector cells frequently lose CD28 expression, but can acquire de novo expression of NK-cell receptors (recognizing MHC class I) and have increased expression of CD70 and LFA-1.32,43,74,75 Such changes could impart NK-cell-like functionality, facilitate interaction with somatic cells and lower T-cell activation thresholds.

\section{The phenotype of RA in the elderly Elderly-onset RA versus young-onset RA}

RA in older people can represent the persistence of a disease that manifests clinically at a younger age (usually before the age of 60 years), referred to as young-onset $\mathrm{RA}$, or a disease that develops at an older age (after 60 years of age), which is referred to as elderly-onset RA. Compared with young-onset RA, elderly-onset RA is characterized by a more balanced gender distribution, a higher frequency of acute onset, and more frequent involvement of large joints; ${ }^{76-79}$ the involvement of the shoulders is often the primary manifestations of elderly-onset RA, complicating its differential diagnosis with polymyalgia rheumatica. Indeed, the disease can initially be diagnosed as polymyalgia and treated with corticosteroid monotherapy, with arthritic symptoms manifesting more clearly upon tapering of the corticosteroid dose. ${ }^{79,80}$ Erythrocyte sedimentation rates and CRP levels are higher, whereas the prevalence of RF is lower in elderly-onset RA than in young-onset RA. ${ }^{76}$
Furthermore, the levels of proinflammatory cytokines differ between young-onset and elderly-onset RA, with higher IL- 6 and lower TNF concentrations observed in the latter. ${ }^{81}$ Whether the disease outcomes are better or worse for elderly-onset RA than young-onset RA remains unclear, and the available evidence is conflicting. ${ }^{82,83}$ These observations demonstrate the complexity of RA pathogenesis and that our understanding of the contribution of ageing of the immune system to this process remains incomplete. Nevertheless, the ageing-related changes in the immune environment described above are likely to influence the pathogenesis and phenotype of elderly-onset RA.

\section{Comorbidities in elderly patients with RA Cardiovascular disease}

Regardless of the age at which RA first manifests, the disease can be associated with comorbidities that complicate the phenotype of the disease. Some of the comorbidities associated with RA might be linked to the autoimmune mechanism underlying the disease, the adverse effects of RA therapies, or a combination of these factors, whereas others might be unrelated either to RA or its treatment. ${ }^{84}$ Mortality rates are higher in the RA population than in the general population, primarily as a result of increased prevalence of ischaemic heart disease and heart failure in the former. ${ }^{85-87} \mathrm{~A}$ population-based study comparing patients with RA and individuals without RA with similar demographics showed that the risk of myocardial infarction leading to hospitalization was 3.17-fold higher in the RA population. ${ }^{86}$ In addition, the incidence of heart failure in patients with RA is approximately twice that observed in individuals without RA. ${ }^{87}$ The increased risk of cardiovascular disease associated with RA seems to be largely confined to patients positive for $\mathrm{RF}^{87}$ Increasing age is a risk factor for ischaemic heart disease, and this relationship might be partially explained by the previously discussed age-related changes in the innate immune system associated with increased levels of proinflammatory cytokines and CRP; ${ }^{88}$ active RA further contributes to this inflammatory environment. Although the increased cardiovascular risk in RA seems to be partially mediated by long-term inflammation, traditional cardiovascular risk factors also have important roles. ${ }^{89}$

\section{Cancer}

The second most common comorbidity causing increased mortality in patients with RA is cancer, ${ }^{84}$ and the risk of cancer is increased in the RA population compared with the general population. ${ }^{90}$ This slightly increased cancer risk is mostly attributable to increased frequencies of a number of specific malignancies, including lymphoma and skin cancer. ${ }^{84}$ Furthermore, Baecklund et al. ${ }^{91}$ showed that the frequency of lymphoma is not only increased in RA but is also related to the severity of the disease. The molecular phenomena underlying the ageing process, and in particular the accumulation of DNA damage, contribute to the risk of cancer. ${ }^{92}$ The elevated cancer risk observed in RA might 
be partially explained by chronic inflammation and by the use of some immunosuppressant drugs, although the relative contributions of the disease itself and the therapy remain a matter of debate. ${ }^{93}$ Regardless of the precise causal relationships between the development of cancer and ageing processes, chronic inflammation and RA therapy, the fact that cancer is prevalent in elderly patients with RA has implications for the management of the rheumatic disease.

\section{Infection}

Patients with RA are at increased risk of infection compared with other individuals from the same population, as a result of the immunomodulatory effects of the disease itself or its associated comorbidities. ${ }^{94}$ Furthermore, the treatments used in RA can also suppress the immune system, leaving the patient at higher risk of infection. ${ }^{95,96}$ Interestingly, age is an independent risk factor for serious infection in patients with RA and, consequently, has been integrated into a new risk score for serious infection in RA. ${ }^{97}$

\section{Venous and arterial insufficiency}

Venous or arterial insufficiency, especially with skin ulceration, is another relevant comorbidity in elderly patients with RA, which can complicate disease management and limit therapeutic options; a retrospective chart analysis of hospitalized patients with RA and leg ulcers found chronic venous insufficiency and/or arterial malfunction to be an important underlying cause of ulceration in this patient group. ${ }^{98}$ Other comorbidities associated with skin ulceration in older patients with RA include ulcerated rheumatoid nodules, necrotic vasculitic lesions, and pyoderma gangrenosum. ${ }^{98}$

\section{Other comorbidities of importance}

Additional comorbidities of special interest in elderly patients with RA-primarily as they affect disease management-include lung disease, diabetes mellitus, gastrointestinal (peptic ulcer) disease, anaemia, osteoporosis, and depression. Furthermore, rheumatoid cachexia-characterized by reduced muscle mass and increased fat mass in individuals with normal or increased body mass indices-might be underrecognized in clinical practice. ${ }^{99}$ Studies of cachexia in RA have revealed no clear single cause of reduced muscle mass, suggesting that this condition is the result of a multifactorial process, with systemic inflammation representing one important contributor. ${ }^{100}$ Rheumatoid cachexia contributes to frailty and to unsuccessful ageing.

Geriatric giant conditions can further be included as important comorbidities that are likely to complicate disease management in elderly patients with RA. Chen et al. ${ }^{101}$ demonstrated that geriatric syndromes such as cognitive impairment, depression, falls, incontinence, and malnutrition are common in elderly individuals with RA and are associated with higher disease activity (28-joint Disease Activity Score [DAS28]), lower haemoglobin levels, and reduced physical independence. In fact, logistic regression analysis revealed that high DAS28 and

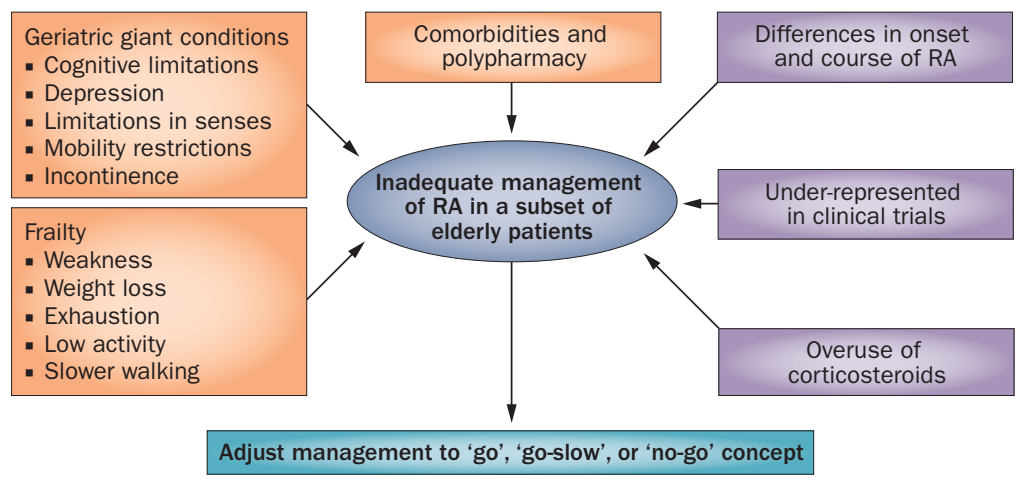

Figure 3 | Challenges facing the management of RA in elderly adults. Management of RA in elderly patients is complicated by many factors. Properties associated with unsuccessful ageing can complicate the management of RA (orange boxes).

Successful ageing can be undermined and treatment complicated by geriatric giant conditions, which limit the patient's independence, as well as by frailty, which reduces the patient's ability to withstand illness without reductions in physical functioning. Frail patients and patients with comorbidities (including cardiovascular disease, cancer, infection) and associated polypharmacy might require a tailored approach to therapy. In addition, factors related to disease pathology or therapy in elderly individuals might pose additional challenges to management of RA in elderly patients (purple boxes). For example, treatment decisions need to take into account differences in the disease characteristics of elderly-onset and young-onset RA, such as the higher expression of IL-6 and the lower expression of TNF in the former. Furthermore, the evidence base for development of treatment recommendations is weak because elderly patients are often excluded from clinical trials. Indeed, DMARDs and biologic agents seem to be under-used in elderly patients with RA, leading to more frequent administration of corticosteroids in this group. A balanced view of the factors influencing the phenotype and management of RA in elderly adults is indispensable for making a 'go', 'go-slow' or 'no-go' decision on treatment. A go-slow approach would make provisions for full DMARD or biologic therapy; in a no-go approach, these therapies would not be used.

low haemoglobin levels were independent risk factors for geriatric giant syndromes. ${ }^{101}$

\section{Polypharmacy}

Comorbidities, such as those typically associated with RA in elderly individuals, are associated with polypharmacy, increasing the risk of iatrogenic effects and complicating disease management. Distinguishing between the effects of a disease and the adverse effects of medication in elderly patients can be challenging; therefore, a thorough medication history should be acquired upon observation of any pathological feature not typically associated with RA or with a known comorbidity.

\section{Management of RA in elderly patients}

Management of RA in elderly individuals is challenging owing to comorbidities and frailty, requires appropriate tailoring of the aggressiveness of the therapeutic approach, and is currently inadequate for several reasons, as discussed in the following paragraphs and summarized in Figure 3. Compared with individuals without RA, life expectancy is reduced by 4 years and 2 years in women and men with RA, respectively, deficits that have not been reduced over the past decades despite overall life expectancy increasing in patients with RA in parallel with the general population. ${ }^{102}$ Therefore, further improvements are needed in the management of elderly individuals with RA. 


\section{Clinical data on treatment effects}

Patient age has been shown to influence decisions made by rheumatologists regarding the agents used to treat RA. ${ }^{103}$ A US study showed that patients with RA $>75$ years of age had a $65 \%$ lower chance of receiving DMARDs than patients aged $65-75$ years, and this difference increased with increasing age (52\% for $75-84$ year olds and $74 \%$ for $>85$ year olds). ${ }^{104}$ Interestingly, the prescription of DMARDs was further reduced in elderly individuals with RA if the treating physician was not a rheumatologist. ${ }^{104}$ In addition, an observational study based on the Consortium of Rheumatology Researchers of North America registry ${ }^{77}$ revealed that both mean methotrexate dose and the proportion of patients receiving multiple DMARD therapy or biologic agents were lower in patients with elderly-onset RA compared with individuals with young-onset RA. Furthermore, a Dutch study ${ }^{105}$ found that younger patients with RA had a substantially higher chance of receiving anti-TNF therapy than older patients within an equal period of time after first indication, despite the eldest patients having the highest DAS28 before treatment.

Thus, existing data suggest that DMARDs and biologic agents are under-used in elderly patients with RA. One possible explanation for this irregularity is that physicians are concerned about the safety and efficacy of DMARDs and biologic agents in elderly adults. However, several studies suggest that these treatments are effective, safe and well tolerated in this patient group. Data from the Dutch Rheumatoid Arthritis Monitoring registry ${ }^{106}$ indicate that treatment of RA with anti-TNF agents markedly reduces disease activity and is well tolerated in all age groups, despite having slightly reduced efficacy in the elderly patients compared with younger individuals. Bathon et al..$^{107}$ reported similar findings, revealing that the efficacy of TNF-blockade using etanercept was lower in elderly patients with RA ( $\geq 65$ years of age) than in younger patients; however, in both age groups, etanercept had a greater therapeutic effect than placebo and was more efficacious in combination with methotrexate than either agent alone. ${ }^{107}$ Furthermore, efficacy responses in elderly individuals were sustained for up to 6 years. ${ }^{107}$ Bathon and colleagues ${ }^{107}$ also reported that, although the rates of serious adverse events after etanercept treatment tend to be higher in elderly patients with RA than in younger individuals with the disease, the rates of adverse events in elderly patients were not substantially different from age-matched patients treated with placebo or methotrexate. Other studies further support the safety and efficacy of etanercept in elderly patients with RA. ${ }^{108,109}$ In addition, Genevay et al. ${ }^{110}$ reported that drug discontinuation rates and mean changes in DAS28-a measure of clinical efficacy-were similar in elderly (again, $\geq 65$ years of age) and younger patients with RA treated with anti-TNF agents, but that HAQ scores-a measure of patient quality of life-improved less in elderly patients, mostly as a consequence of higher baseline HAQ scores (and thus lower quality of life) in patients $>75$ years of age.

Risk of serious infection is a particular concern both in users of immunomodulatory agents (independent of age) and in elderly individuals. Nevertheless, an observational study using data from the British Society for Rheumatology Biologics Register ${ }^{111}$ confirmed that the relative risk of infection associated with immunosuppressive anti-TNF therapy is not increased in older patients with RA; although the relative risk of infection was similar in all age groups, the absolute risk of infection was higher in patients $>65$ years of age treated with anti-TNF therapy than in younger patients. Similarly, Schneeweiss et al. ${ }^{12}$ reported that the risk of serious bacterial infections in elderly patients with RA did not differ between patients receiving anti-TNF agents and those receiving methotrexate, although the risk was dosedependently increased by glucocorticoid usage. Together, these studies demonstrate that therapeutic regimens that are effective and well tolerated in younger adults are also efficacious and have an acceptable safety profile in elderly patients, and thus could be integrated into the therapeutic management of elderly patients with RA.

\section{Tailoring therapy}

In elderly patients with RA, the target and choice of therapy should be tailored according to disease activity and severity, risk factors for adverse effects of drugs, comorbidities and the patient's general vitality versus frailty (Figure 3). The level of disease activity set as the target for therapy might differ according to biological age or owing to the presence of particular risk factors for infection. The challenge is to define which patients will benefit from aggressive therapy, opposed to those who are less likely to benefit and could be negatively affected by such an approach, in whom therapeutic aggressiveness should thus be tapered down.

These factors must be integrated into a management strategy and can be helpful in deciding whether to take a 'go', 'go-slow', or 'no-go' approach to intervention with a particular therapeutic. For example, an 80-yearold person with RA who is otherwise in generally good health, lives independently, and regularly exercises and participates in social activities would qualify as a 'go', owing to good vitality and low frailty, and thus a good likelihood of tolerance of and response to therapy. On the other hand, a person of the same age who has aged less successfully (has a higher biological age), is in bad general health, and has multiple comorbidities and minimal participation in social activities would probably be better managed with a go-slow approach or, in certain situations, a no-go approach. A 'go-slow' profile would not rule out the use of full DMARD or biologic therapy, but would necessitate careful consideration of the conditions of their use. A 'no-go' profile does not mean withholding necessary measures to support the patient's comfort; however, for this category of patients, alternative approaches replacing more aggressive therapeutic strategies might be considered and full DMARD and biologic therapies should probably not be used. The challenges in the 'go, go-slow, no-go' approach to patient management lie in accurately assessing patient profiles. A general concern relating to the developing management strategies is the lack of clinical trial data in elderly patients, which necessitates further 
study. This problem faces many areas of medicine, including fields in which ageing is of particular relevance, such as rheumatology and oncology. ${ }^{11,114}$

\section{Conclusions}

Ageing is associated with profound changes in the body's immune milieu. These changes can influence both the onset and clinical phenotype of RA. Indications are that some elderly patients with RA are currently undertreated, and more ambitious targets could be reached in many patients. However, therapeutic decisions in these patients are complicated by comorbidities, especially cardiovascular disease, cancer and infection, which are associated with both RA and ageing. When making treatment decisions, concepts of biological age and successful versus unsuccessful ageing must be considered. Although observational studies suggest that DMARDs and biologic agents are effective and well tolerated in elderly patients with RA, additional clinical studies are needed in which the treatment regimen is tailored according to parameters of vitality or frailty.

Review criteria
MEDLINE and PubMed databases were searched
for original articles published between 2000 and
December 2012 focusing on mechanisms of ageing,
immunosenescence, and phenotype and management of
rheumatoid arthritis in the elderly. The search terms used
in combination with "rheumatoid arthritis" were "ageing",
"elderly", "treatment", "comorbidity". Additional search
terms used were "elderly-onset rheumatoid arthritis",
"immunosenescence", "frailty". The reference lists of
identified articles were also searched for further papers
of relevance.

1. Konrat, C. et al. Underrepresentation of elderly people in randomised controlled trials. The example of trials of 4 widely prescribed drugs. PLOS ONE 7, e33559 (2012).

2. Macieira-Coelho, A. Cell division and aging of the organism. Biogerontology 12, 503-515 (2011).

3. Sperka, T., Wang, J. \& Rudolph, K. L. DNA damage checkpoints in stem cells, ageing and cancer. Nat. Rev. Mol. Cell. Biol. 13, 579-590 (2012).

4. Kirkwood, T. B. Evolution of ageing. Nature $\mathbf{2 7 0}$ 301-304 (1977).

5. Kirkwood, T. L., Kapahi, P. \& Shanley, D. P. Evolution, stress, and longevity. J. Anat. 197, 587-590 (2000).

6. Ljubuncic, P. \& Reznick, A. Z. The evolutionary theories of aging revisited-a mini-review. Gerontology 55, 205-216 (2009).

7. Fulop, T. et al. Aging, frailty and age-related diseases. Biogerontology 11, 547-563 (2010).

8. Isaacs, B. The challenge of geriatric medicine (Oxford University Press, 1997).

9. Health Council of the Netherlands. Health care for the elderly with multimorbidity. Publication no. 2008/01 (Health Council of the Netherlands, 2008).

10. Lang, P. O., Michel, J. P. \& Zekry, D. Frailty syndrome: a transitional state in a dynamic process. Gerontology 55, 539-549 (2009).

11. Fried, L. P. et al. Frailty in older adults: evidence for a phenotype. J. Gerontol. A Biol. Sci. Med. Sci. 56, M146-156 (2001).

12. Bandeen-Roche, $K$. et al. Phenotype of frailty: characterization in the women's health and aging studies. J. Gerontol. A Biol. Sci. Med. Sci. 61, 262-266 (2006)

13. Garonzik-Wang, J. M. et al. Frailty and delayed graft function in kidney transplant recipients. Arch. Surg. 147, 190-193 (2012).

14. Makary, M. A. et al. Frailty as a predictor of surgical outcomes in older patients. J. Am. Coll. Surg. 210, 901-908 (2010).

15. Yao, X. et al. Frailty is associated with impairment of vaccine-induced antibody response and increase in post-vaccination influenza infection in community-dwelling older adults. Vaccine 29, 5015-5021 (2011)

16. Abadir, P. M. et al. Identification and characterization of a functional mitochondrial angiotensin system. Proc. Natl Acad. Sci. USA 108, 14849-14854 (2011).

17. Leng, S. X., Yang, H. \& Walston, J. D. Decreased cell proliferation and altered cytokine production in frail older adults. Aging Clin. Exp. Res. 16, 249-252 (2004)
18. Varadhan, R. et al. Higher levels and blunted diurnal variation of cortisol in frail older women. J. Gerontol. A Biol. Sci. Med. Sci. 63, 190-195 (2008).

19. Gameiro, C. \& Romao, F. Changes in the immune system during menopause and aging. Front. Biosci. (Elite Ed.) 2, 1299-1303 (2010).

20. Gomez, C. R., Boehmer, E. D. \& Kovacs, E. J. The aging innate immune system. Curr. Opin. Immunol. 17, 457-462 (2005).

21. Krabbe, K. S., Pedersen, M. \& Bruunsgaard, H. Inflammatory mediators in the elderly. Exp. Gerontol. 39, 687-699 (2004).

22. Panda, A. et al. Human innate immunosenescence: causes and consequences for immunity in old age. Trends Immunol. $\mathbf{3 0}$, 325-333 (2009).

23. Shaw, A. C., Joshi, S., Greenwood, H., Panda, A. \& Lord, J. M. Aging of the innate immune system. Curr. Opin. Immunol. 22, 507-513 (2010).

24. Goronzy, J. J., Shao, L. \& Weyand, C. M. Immune aging and rheumatoid arthritis. Rheum. Dis. Clin. North Am. 36, 297-310 (2010).

25. Lindstrom, T. M. \& Robinson, W. H. Rheumatoid arthritis: a role for immunosenescence? J. Am. Geriatr. Soc. 58, 1565-1575 (2010).

26. Scullion, J. Tuberculosis and older people. Nurs. Older People 15, 23-27 (2003).

27. Frasca, D. \& Blomberg, B. B. Aging affects human B cell responses. J. Clin. Immunol. 31, 430-435 (2011).

28. Lee, N., Shin, M. S. \& Kang, I. T-cell biology in aging, with a focus on lung disease. J. Gerontol. A Biol. Sci. Med. Sci. 67, 254-263 (2012).

29. Hakim, F. T. et al. Age-dependent incidence, time course, and consequences of thymic renewal in adults. J. Clin. Invest. 115, 930-939 (2005).

30. Geginat, J., Lanzavecchia, A. \& Sallusto, F. Proliferation and differentiation potential of human $\mathrm{CD}^{+}$memory T-cell subsets in response to antigen or homeostatic cytokines. Blood 101, 4260-4266 (2003).

31. Broux, B., Markovic-Plese, S., Stinissen, P. \& Hellings, N. Pathogenic features of $\mathrm{CD} 4{ }^{+} \mathrm{CD} 28$ $\mathrm{T}$ cells in immune disorders. Trends Mol. Med. 18, 446-453 (2012).

32. Weng, N. P., Akbar, A. N. \& Goronzy, J. CD28$T$ cells: their role in the age-associated decline of immune function. Trends Immunol. $\mathbf{3 0}$, 306-312 (2009).

33. Vallejo, A. N. et al. Molecular basis for the loss of CD28 expression in senescent T cells. J. Biol. Chem. 277, 46940-46949 (2002).
34. Valenzuela, H. F. \& Effros, R. B. Divergent telomerase and CD28 expression patterns in human CD4 and CD8 T cells following repeated encounters with the same antigenic stimulus. Clin. Immunol. 105, 117-125 (2002).

35. Koch, S. et al. Cytomegalovirus infection: a driving force in human T cell immunosenescence. Ann. NY Acad. Sci. 1114 23-35 (2007).

36. van Leeuwen, E. M. et al. Emergence of a $\mathrm{CD}^{+} \mathrm{CD} 28^{-}$granzyme $\mathrm{B}^{+}$, cytomegalovirusspecific $T$ cell subset after recovery of primary cytomegalovirus infection. J. Immunol. 173, 1834-1841 (2004).

37. Schirmer, M., Vallejo, A. N., Weyand, C. M. \& Goronzy, J. J. Resistance to apoptosis and elevated expression of $\mathrm{Bcl}-2$ in clonally expanded $C D 4^{+} \mathrm{CD} 28^{-} \mathrm{T}$ cells from rheumatoid arthritis patients. J. Immunol. 161, 1018-1025 (1998).

38. Vallejo, A. N., Schirmer, M., Weyand, C. M. \& Goronzy, J. J. Clonality and longevity of $\mathrm{CD} 4{ }^{+} \mathrm{CD} 28^{\text {null }} \mathrm{T}$ cells are associated with defects in apoptotic pathways. J. Immunol. 165, 6301-6307 (2000).

39. Vallejo, A. N. et al. Expansions of NK-like $\alpha \beta T$ cells with chronologic aging: novel lymphocyte effectors that compensate for functional deficits of conventional NK cells and T cells. Ageing Res. Rev. 10, 354-361 (2011).

40. Heyn, H. et al. Distinct DNA methylomes of newborns and centenarians. Proc. Natl Acad. Sci. USA 109, 10522-10527 (2012).

41. Liu, Y., Chen, Y. \& Richardson, B. Decreased DNA methyltransferase levels contribute to abnormal gene expression in "senescent" CD4 ${ }^{+}$CD28- T cells. Clin. Immunol. 132 , 257-265 (2009).

42. Schmidt, D., Martens, P. B., Weyand, C. M. \& Goronzy, J. J. The repertoire of CD4 ${ }^{+} \mathrm{CD}^{28}$ T cells in rheumatoid arthritis. Mol. Med. 2, 608-618 (1996).

43. Schmidt, D., Goronzy, J. J. \& Weyand, C. M. $\mathrm{CD}^{+}{ }^{+} \mathrm{CD} 7^{-} \mathrm{CD} 28^{-} \mathrm{T}$ cells are expanded in rheumatoid arthritis and are characterized by autoreactivity. J. Clin. Invest. 97, 2027-2037 (1996).

44. Bristol-Myers Squibb. Abatacept summary of product characteristics [online], http:// www.ema.europa.eu/docs/en GB/ document_library/EPAR__Product_Information/ human/000701/WC500048935.pdf

45. Kremer, J. M. et al. Treatment of rheumatoid arthritis by selective inhibition of T-cell activation 
with fusion protein CTLA4lg. N. Engl. J. Med. 349, 1907-1915 (2003).

46. Arai, Y., Takayama, M., Abe, Y. \& Hirose, N. Adipokines and aging. J. Atheroscler. Thromb. 18, 545-550 (2011).

47. Conde, J. et al. Adipokines: novel players in rheumatic diseases. Discov. Med. 15, 73-83 (2013).

48. Gomez, R. et al. What's new in our understanding of the role of adipokines in rheumatic diseases? Nat. Rev. Rheumatol. 7 , 528-536 (2011).

49. Olama, S. M., Senna, M. K. \& Elarman, M. Synovial/serum leptin ratio in rheumatoid arthritis: the association with activity and erosion. Rheumatol. Int. 32, 683-690 (2012).

50. Matarese, G., Procaccini, C., De Rosa, V., Horvath, T. L. \& La Cava, A. Regulatory T cells in obesity: the leptin connection. Trends Mol. Med. 16, 247-256 (2010).

51. Giles, J. T., Allison, M., Bingham, C. O. $3^{\text {rd }}$, Scott, W. M. Jr \& Bathon, J. M. Adiponectin is a mediator of the inverse association of adiposity with radiographic damage in rheumatoid arthritis. Arthritis Rheum. 61, 1248-1256 (2009).

52. Matthews, S. J. \& Lancaster, J. W. Urinary tract infections in the elderly population. Am. J. Geriatr. Pharmacother. 9, 286-309 (2011).

53. Meyer, K. C. The role of immunity and inflammation in lung senescence and susceptibility to infection in the elderly. Semin. Respir. Crit. Care Med. 31, 561-574 (2010).

54. Mori, T. \& Leung, C. C. Tuberculosis in the global aging population. Infect. Dis. Clin. North Am. 24, 751-768 (2010).

55. Jefferson, T. et al. Efficacy and effectiveness of influenza vaccines in elderly people: a systematic review. Lancet 366, 1165-1174 (2005).

56. Kumar, R. \& Burns, E. A. Age-related decline in immunity: implications for vaccine responsiveness. Expert. Rev. Vaccines 7, 467-479 (2008).

57. Lang, P. O., Govind, S., Mitchell, W. A., Siegrist, C. A. \& Aspinall, R. Vaccine effectiveness in older individuals: what has been learned from the influenza-vaccine experience. Ageing Res. Rev. 10, 389-395 (2011).

58. Lang, P. O. et al. Effectiveness of influenza vaccine in aging and older adults: comprehensive analysis of the evidence. Clin. Interv. Aging 7, 55-64 (2012).

59. McElhaney, J. E. Influenza vaccine responses in older adults. Ageing Res. Rev. 10, 379-388 (2011).

60. Fujihashi, K. \& Kiyono, H. Mucosal immunosenescence: new developments and vaccines to control infectious diseases. Trends Immunol. 30, 334-343 (2009).

61. Giuliano, S., Ohanna, M., Ballotti, R. \& Bertolotto, C. Advances in melanoma senescence and potential clinical application. Pigment Cell Melanoma Res. 24, 295-308 (2011).

62. Lasithiotakis, K. G., Petrakis, I. E. \& Garbe, C. Cutaneous melanoma in the elderly: epidemiology, prognosis and treatment. Melanoma Res. 20, 163-170 (2010).

63. Finkel, T., Serrano, M. \& Blasco, M. A. The common biology of cancer and ageing. Nature 448, 767-774 (2007).

64. de Vlam, K. et al. Detection and identification of antinuclear autoantibodies in the serum of normal blood donors. Clin. Exp. Rheumatol. 11, 393-397 (1993).

65. Borchers, A. T. \& Gershwin, M. E. Giant cell arteritis: a review of classification, pathophysiology, geoepidemiology and treatment. Autoimmun. Rev. 11, A544-A554 (2012).
66. Crowson, C. S. et al. The lifetime risk of adultonset rheumatoid arthritis and other inflammatory autoimmune rheumatic diseases. Arthritis Rheum. 63, 633-639 (2011).

67. Fujii, H., Shao, L., Colmegna, I., Goronzy, J. J. \& Weyand, C. M. Telomerase insufficiency in rheumatoid arthritis. Proc. Natl Acad. Sci. USA 106, 4360-4365 (2009).

68. Weyand, C. M., Fujii, H., Shao, L. \& Goronzy, J. J. Rejuvenating the immune system in rheumatoid arthritis. Nat. Rev. Rheumatol. 5, 583-588 (2009).

69. Koetz, K. et al. T cell homeostasis in patients with rheumatoid arthritis. Proc. Nat/ Acad. Sci. USA 97, 9203-9208 (2000).

70. Goronzy, J. J. \& Weyand, C. M. Immune aging and autoimmunity. Cell. Mol. Life Sci. 69, 1615-1623 (2012).

71. Colmegna, I. et al. Defective proliferative capacity and accelerated telomeric loss of hematopoietic progenitor cells in rheumatoid arthritis. Arthritis Rheum. 58, 990-1000 (2008).

72. Shao, L. et al. Deficiency of the DNA repair enzyme ATM in rheumatoid arthritis. J. Exp. Med. 206, 1435-1449 (2009).

73. Kohler, S. \& Thiel, A. Life after the thymus: $\mathrm{CD} 1^{+}$and $\mathrm{CD} 31^{-}$human naive $\mathrm{CD} 4^{+}{ }^{-}$-cell subsets. Blood 113, 769-774 (2009).

74. Lee, W. W., Yang, Z. Z., Li, G., Weyand, C. M. \& Goronzy, J. J. Unchecked CD70 expression on $T$ cells lowers threshold for $T$ cell activation in rheumatoid arthritis. J. Immunol. 179, 2609-2615 (2007).

75. Singh, K., Colmegna, I., He, X., Weyand, C. M. \& Goronzy, J. J. Synoviocyte stimulation by the LFA-1-intercellular adhesion molecule-2-EzrinAkt pathway in rheumatoid arthritis. J. Immunol. 180, 1971-1978 (2008).

76. Mavragani, C. P. \& Moutsopoulos, H. M. Rheumatoid arthritis in the elderly. Exp. Gerontol. 34, 463-471 (1999).

77. Tutuncu, Z. \& Kavanaugh, A. Rheumatic disease in the elderly: rheumatoid arthritis. Clin. Geriatr. Med. 21, 513-525 (2005).

78. van Schaardenburg, D. \& Breedveld, F. C. Elderlyonset rheumatoid arthritis. Semin. Arthritis Rheum. 23, 367-378 (1994).

79. Villa-Blanco, J. I. \& Calvo-Alen, J. Elderly onset rheumatoid arthritis: differential diagnosis and choice of first-line and subsequent therapy. Drugs Aging 26, 739-750 (2009).

80. Kermani, T. A. \& Warrington, K. J. Polymyalgia rheumatica. Lancet 381, 63-72 (2013).

81. Chen, D. Y. et al. Proinflammatory cytokine profiles of patients with elderly-onset rheumatoid arthritis: a comparison with youngeronset disease. Gerontology 55, 250-258 (2009).

82. Glennas, A., Kvien, T. K., Andrup, O., Karstensen, B. \& Munthe, E. Recent onset arthritis in the elderly: a 5 year longitudinal observational study. J. Rheumatol. 27, 101-108 (2000).

83. van der Heijde, D. M. et al. Older versus younger onset rheumatoid arthritis: results at onset and after 2 years of a prospective followup study of early rheumatoid arthritis. J. Rheumatol. 18, 1285-1289 (1991)

84. Gabriel, S. E. \& Michaud, K. Epidemiological studies in incidence, prevalence, mortality, and comorbidity of the rheumatic diseases. Arthritis Res. Ther. 11, 229 (2009).

85. Maradit-Kremers, H. et al. Increased unrecognized coronary heart disease and sudden deaths in rheumatoid arthritis: a population-based cohort study. Arthritis Rheum. 52, 402-411 (2005).

86. Maradit-Kremers, H., Nicola, P. J., Crowson, C. S., Ballman, K. V. \& Gabriel, S. E.
Cardiovascular death in rheumatoid arthritis: a population-based study. Arthritis Rheum. $\mathbf{5 2}$ 722-732 (2005).

87. Nicola, P. J. et al. The risk of congestive heart failure in rheumatoid arthritis: a populationbased study over 46 years. Arthritis Rheum. 52, 412-420 (2005).

88. Madjid, M. \& Willerson, J. T. Inflammatory markers in coronary heart disease. Br. Med. Bull. 100, 23-38 (2011)

89. Liao, K. P. \& Solomon, D. H. Traditional cardiovascular risk factors, inflammation and cardiovascular risk in rheumatoid arthritis. Rheumatology (Oxford) 52, 45-52 (2013).

90. Smitten, A. L., Simon, T. A., Hochberg, M. C. \& Suissa, S. A meta-analysis of the incidence of malignancy in adult patients with rheumatoid arthritis. Arthritis Res. Ther. 10, R45 (2008).

91. Baecklund, E. et al. Association of chronic inflammation, not its treatment, with increased lymphoma risk in rheumatoid arthritis. Arthritis Rheum. 54, 692-701 (2006).

92. Niccoli, T. \& Partridge, L. Ageing as a risk factor for disease. Curr. Biol. 22, R741-R752 (2012).

93. Askling, J. \& Bongartz, T. Malignancy and biologic therapy in rheumatoid arthritis. Curr. Opin. Rheumatol. 20, 334-339 (2008).

94. Doran, M. F., Crowson, C. S., Pond, G. R., O'Fallon, W. M. \& Gabriel, S. E. Frequency of infection in patients with rheumatoid arthritis compared with controls: a population-based study. Arthritis Rheum. 46, 2287-2293 (2002).

95. De Keyser, F. Choice of biologic therapy for patients with rheumatoid arthritis: the infection perspective. Curr. Rheumatol. Rev. 7, 77-87 (2011)

96. Salliot, C., Dougados, M. \& Gossec, L. Risk of serious infections during rituximab, abatacept and anakinra treatments for rheumatoid arthritis: meta-analyses of randomised placebocontrolled trials. Ann. Rheum. Dis. 68, 25-32 (2009).

97. Crowson, C. S., Hoganson, D. D., FitzGibbon, P. D. \& Matteson, E. L. Development and validation of a risk score for serious infection in patients with rheumatoid arthritis. Arthritis Rheum. 64, 2847-2855 (2012).

98. Seitz, C. S., Berens, N., Brocker, E. B. \& Trautmann, A. Leg ulceration in rheumatoid arthritis - an underreported multicausal complication with considerable morbidity: analysis of thirty-six patients and review of the literature. Dermatology 220, 268-273 (2010).

99. Summers, G. D., Deighton, C. M., Rennie, M. J. \& Booth, A. H. Rheumatoid cachexia: a clinical perspective. Rheumatology (Oxford) 47, 1124-1131 (2008).

100. Munro, R. \& Capell, H. Prevalence of low body mass in rheumatoid arthritis: association with the acute phase response. Ann. Rheum. Dis. 56 326-329 (1997).

101. Chen, Y. M., Chen, L. K., Lan, J. L. \& Chen, D. Y. Geriatric syndromes in elderly patients with rheumatoid arthritis. Rheumatology (Oxford) 48, 1261-1264 (2009).

102. Ziade, N., Jougla, E. \& Coste, J. Population-level influence of rheumatoid arthritis on mortality and recent trends: a multiple cause-of-death analysis in France, 1970-2002. J. Rheumatol. 35, 1950-1957 (2008).

103. Fraenkel, L., Rabidou, N. \& Dhar, R. Are rheumatologists' treatment decisions influenced by patients' age? Rheumatology (Oxford) $\mathbf{4 5}$, 1555-1557 (2006).

104. Schmajuk, G. et al. Treatment of older adult patients diagnosed with rheumatoid arthritis: improved but not optimal. Arthritis Rheum. 57, 928-934 (2007). 
105. Radovits, B. J., Fransen, J., Eijsbouts, A. van Riel, P. L. \& Laan, R. F. Missed opportunities in the treatment of elderly patients with rheumatoid arthritis. Rheumatology (Oxford) 48, 906-910 (2009).

106. Radovits, B. J. et al. Influence of age on the outcome of antitumour necrosis factor alpha therapy in rheumatoid arthritis. Ann. Rheum. Dis. 68, 1470-1473 (2009).

107. Bathon, J. M. et al. Safety and efficacy of etanercept treatment in elderly subjects with rheumatoid arthritis. J. Rheumatol. 33, 234-243 (2006).

108. Fleischmann, R. M. et al. Response to etanercept (Enbrel) in elderly patients with rheumatoid arthritis: a retrospective analysis of clinical trial results. J. Rheumatol. 30, 691-696 (2003).

109. Lurati, A., Marrazza, M., Angela, K. \& Scarpellini, M. Safety of etanercept in elderly subjects with rheumatoid arthritis. Biologics 4, 1-4 (2010).
110. Genevay, S. et al. Tolerance and effectiveness of anti-tumor necrosis factor alpha therapies in elderly patients with rheumatoid arthritis: a population-based cohort study. Arthritis Rheum. 57, 679-685 (2007).

111. Galloway, J. B. et al. Anti-TNF therapy is associated with an increased risk of serious infections in patients with rheumatoid arthritis especially in the first 6 months of treatment: updated results from the British Society for Rheumatology Biologics Register with special emphasis on risks in the elderly. Rheumatology (Oxford) 50, 124-131 (2011).

112. Schneeweiss, S. et al. Anti-tumor necrosis factor alpha therapy and the risk of serious bacterial infections in elderly patients with rheumatoid arthritis. Arthritis Rheum. 56, 1754-1764 (2007).

113. Dotan, E., Browner, I., Hurria, A. \& Denlinger, C. Challenges in the management of older patients with colon cancer. J. Natl Compr. Canc. Netw. 10, 213-224 (2012).

114. Pallis, A. G. \& Scarci, M. Are we treating enough elderly patients with early stage non-small cell lung cancer? Lung Cancer 74, 149-154 (2011).

Acknowledgements

The inspiration for this article came from the Academy of Immunology for Clinicians, Belgium, Spring Lecture Sessions on ageing and immune-mediated

inflammatory disorders. The authors thank $\mathrm{H}$. Van de Keere (10A, Maldegem, Belgium) and P. Leventhal (4Clinics, Paris, France) for English-language editorial assistance.

\section{Author contributions}

All authors contributed to researching data for the article, discussion of content and review/editing of the manuscript before submission. F. De Keyser wrote the article. 